Preprint typeset in JHEP style - HYPER VERSION

\title{
Effective dynamics of the closed loop quantum cosmology
}

\author{
Jakub Mielczarek ${ }^{a *}$ Orest Hrycyna ${ }^{b \dagger}$ and Marek Szydłowski ${ }^{a b c \ddagger}$ \\ a Astronomical Observatory, Jagiellonian University, \\ 30-244 Kraków, Orla 171, Poland \\ ${ }^{b}$ Department of Theoretical Physics, Catholic University of Lublin, \\ Al. Ractawickie 14, 20-950 Lublin, Poland \\ ${ }^{c}$ Marc Kac Complex Systems Research Centre, \\ Jagiellonian University, Reymonta 4, 30-059 Kraków, Poland
}

\begin{abstract}
In this paper we study dynamics of the closed FRW model with holonomy corrections coming from loop quantum cosmology. We consider models with a scalar field and cosmological constant. In case of the models with cosmological constant and free scalar field, dynamics reduce to $2 \mathrm{D}$ system and analysis of solutions simplify. If only free scalar field is included then universe undergoes non-singular oscillations. For the model with cosmological constant, different behaviours are obtained depending on the value of $\Lambda$. If the value of $\Lambda$ is sufficiently small, bouncing solutions with asymptotic de Sitter stages are obtained. However if the value of $\Lambda$ exceeds critical value $\Lambda_{\mathrm{c}}=\frac{\sqrt{3} m_{\mathrm{P} 1}^{2}}{2 \pi \gamma^{3}} \simeq 21 m_{\mathrm{Pl}}^{2}$ then solutions become oscillatory. Subsequently we study models with a massive scalar field. We find that this model possess generic inflationary attractors. In particular field, initially situated in the bottom of the potential, is driven up during the phase of quantum bounce. This subsequently leads to the phase of inflation. Finally we find that, comparing with the flat case, effects of curvature do not change qualitatively dynamics close to the phase of bounce. Possible effects of inverse volume corrections are also briefly discussed.
\end{abstract}

\footnotetext{
*jakub.mielczarek@uj.edu.pl

†hrycyna@kul.lublin.pl

${ }_{\ddagger}^{\ddagger}$ uoszydlo@cyf-kr.edu.pl
} 


\section{Contents}

1. Introduction 1

2. Effective equations for the closed FRW cosmology 3

3. Models with $\Lambda$ and free scalar field as 2D dynamical systems 6

3.1 Cosmological constant 6

3.2 Free scalar field 8

4. Qualitative analysis of the model with quadratic potential function 10

5. Remarks on inverse volume corrections

6. Summary 15

\section{Introduction}

In the recent years methods of the Loop Quantum Gravity (LQG) [1] have been successfully applied to quantise cosmological minisuperspace models. General prediction of this approach, called Loop Quantum Cosmology (LQC) [2], is avoidance of the initial singularity. Namely in LQC singularity is replaced by the phase of bounce. This result seems to be generic for the homogeneous cosmologies and was confirmed in the numerous investigations. Loop quantisation of the homogeneous and isotropic FRW models have been performed in Ref. [3, 田, 5] $(K=0)$,

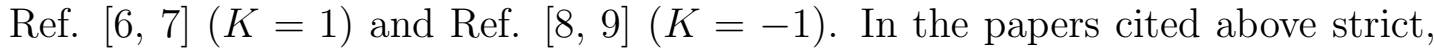
fully quantum considerations were performed. However models only with a free scalar field and cosmological constant were studied.

Dynamics of the models also can be traced with the effective equations of motion. Then classical dynamics is modified with certain nonperturbative quantum gravitational corrections. These corrections becomes unimportant for the low energy densities leading to the classical equations of motion. However for the scales comparable with the Planck energy densities corrections produce effective 
"quantum repulsion" leading to the bounce. Studies of the effective dynamics have recently attracted substantial interest. It is mainly due to the fact that it gives the access to studying various physical consequences of the loop quantisation. Moreover, semi-classical considerations are in many points simpler than the fully quantum treatment. Often approximate solution can be found before the strict quantum results are obtained. In particular models with self-interacting scalar field can be easily studied in this effective formulation. Therefore both fully quantum and effective methods are complementary to each other. Since now effective flat FRW [10, 11] and Bianchi I [12] models in LQC were studied. In the present paper we study effective dynamics of the closed $(K=1)$ FRW model.

We have to stress that alternative effective description is developed by Bojowald and his collaborators. In the Bojowald's approach information about dispersions and higher moments of the observables can also be obtained. Recently this approach has been applied to Bianchi I model [13]. However we do not apply this approach in our considerations. Recently also new approach to quantise loop quantum cosmological models has been proposed [14, 15]. This formulation leads to the bouncing solutions but no effects of so called Thiemann's term, which leads to inverse volume corrections, are present. Obtained effective dynamics is therefore the same like this studied in [10, 11] where only holonomy corrections were taken into account.

Canonical variables for the closed FRW model are similar like in the flat case. Namely original Ashtekar variables $\left(A_{a}^{i}, E_{i}^{a}\right)$ are parametrised by the conjugated $(c, p)$ variables with the Poisson bracket

$$
\{c, p\}=\frac{8 \pi G \gamma}{3}
$$

Parameters $(c, p)$ can be expressed in terms of the standard FRW variables in the following way

$$
p=a^{2}\left(\frac{l_{0}}{a_{0}}\right)^{2} \quad \text { and } c=\gamma \dot{a} \frac{l_{0}}{a_{0}}
$$

where $a_{0}=2$, fiducial volume $V_{0}=2 \pi^{2} a_{0}^{3}=16 \pi^{2}$ and $l_{0}=V_{0}^{1 / 3}=\left(16 \pi^{2}\right)^{1 / 3}=$ $\left(2 \pi^{2}\right)^{1 / 3} a_{0}$. It will become useful when correspondence with classical case will be studied.

In this paper value of Barbero-Immirzi parameter $\gamma=0.2375$, following Ref. [16], is assumed. We also use notation $\kappa=8 \pi G$. 


\section{Effective equations for the closed FRW cosmology}

Starting point of our considerations is the effective Hamiltonian

$$
\mathcal{H}_{\mathrm{eff}}=\frac{-3 \sqrt{p} N}{8 \pi G \gamma^{2} \bar{\mu}^{2}}\left[\sin ^{2}\left(\bar{\mu} c-\bar{\mu} \frac{l_{0}}{2}\right)-\sin ^{2}\left(\bar{\mu} \frac{l_{0}}{2}\right)+\left(1+\gamma^{2}\right) \frac{\bar{\mu}^{2} l_{0}^{2}}{4}\right]+\mathcal{H}_{\mathrm{m}}
$$

which was derived in Ref. [6]. Here $\bar{\mu}$ is quantisation parameter and its form

depends on the particular quantisation scheme. In this paper we choose so called $\bar{\mu}$-scheme, then

$$
\bar{\mu}=\sqrt{\frac{\Delta}{p}}
$$

where $\Delta=2 \sqrt{3} \pi \gamma l_{\mathrm{Pl}}^{2}$. Earlier, $\mu_{0}$-scheme was also used, however it was realised later that it has wrong classical limit. Recently other quantisation scheme was proposed [17] and studied in Ref. [18, 19]. In this scheme new types of finite scale factor singularities appears. Another possibility is those presented in [14, 15] where $\bar{\mu}=\lambda / \sqrt{p}$ and $\lambda$ is some unknown constant unrelated with $\Delta$.

As a matter content we consider homogeneous scalar field with the Hamiltonian

$$
\mathcal{H}_{\mathrm{m}}=N p^{3 / 2}\left(\frac{p_{\phi}^{2}}{2 p^{3}}+V(\phi)\right) .
$$

With this assumption it will be possible to study realisation of the inflationary phase in the bouncing universe. Energy density of the scalar field can be derived from the following expression

$$
\rho:=\frac{1}{p^{3 / 2}} \frac{\partial \mathcal{H}_{\mathrm{m}}}{\partial N} .
$$

Since the Hamiltonian constraint $\frac{\partial \mathcal{H}_{\text {eff }}}{\partial N}=0$ is fulfilled we obtain

$$
\sin ^{2}\left(\bar{\mu} c-\bar{\mu} \frac{l_{0}}{2}\right)=\left[\sin ^{2}\left(\bar{\mu} \frac{l_{0}}{2}\right)-\left(1+\gamma^{2}\right) \frac{\bar{\mu}^{2} l_{0}^{2}}{4}\right]+\frac{\rho}{\rho_{c}}
$$

where we have defined critical density

$$
\rho_{\mathrm{c}}=\frac{3}{\kappa \gamma^{2} \Delta}=\frac{\sqrt{3}}{16 \pi^{2} \gamma^{3} l_{\mathrm{Pl}}^{4}} \simeq 0.82 \rho_{\mathrm{Pl}} .
$$

In the rest of the paper we choose the gauge $N=1$. 
The equations of motion can be derived with the use of Hamilton equation

$$
\dot{f}=\left\{f, \mathcal{H}_{\text {eff }}\right\}
$$

where the Poisson bracket is defined as follows

$$
\{f, g\}=\frac{8 \pi G \gamma}{3}\left[\frac{\partial f}{\partial c} \frac{\partial g}{\partial p}-\frac{\partial f}{\partial p} \frac{\partial g}{\partial c}\right]+\left[\frac{\partial f}{\partial \phi} \frac{\partial g}{\partial p_{\phi}}-\frac{\partial f}{\partial p_{\phi}} \frac{\partial g}{\partial \phi}\right] .
$$

From this definition we can retrieve the elementary brackets

$$
\{c, p\}=\frac{8 \pi G \gamma}{3} \text { and }\left\{\phi, p_{\phi}\right\}=1 .
$$

Based on Eq. 2.7 we derive equation for the scalar field part

$$
\begin{aligned}
\dot{\phi} & =\left\{\phi, \mathcal{H}_{\mathrm{eff}}\right\}=p^{-3 / 2} p_{\phi}, \\
\dot{p_{\phi}} & =\left\{p_{\phi}, \mathcal{H}_{\mathrm{eff}}\right\}=-p^{3 / 2} \frac{d V}{d \phi}
\end{aligned}
$$

what leads to the classical equation

$$
\ddot{\phi}+\frac{3}{2} \frac{\dot{p}}{p} \dot{\phi}+\frac{d V}{d \phi}=0 .
$$

Here equation of motion for the scalar fields does not feel the quantum corrections.

For the gravity part we calculate

$$
\dot{p}=\left\{p, \mathcal{H}_{\mathrm{eff}}\right\}=-\frac{8 \pi G \gamma}{3} \frac{\partial \mathcal{H}_{\mathrm{eff}}}{\partial c}
$$

what together with Eq. 2.5 give us effective Friedmann equation

$$
H^{2}:=\left(\frac{\dot{p}}{2 p}\right)^{2}=\frac{8 \pi G}{3} \frac{1}{\rho_{\mathrm{c}}}\left(\rho-\rho_{1}(p)\right)\left(\rho_{2}(p)-\rho\right)
$$

where

$$
\begin{aligned}
& \rho_{1}(p)=-\rho_{\mathrm{c}}\left[\sin ^{2}\left(\sqrt{\frac{\Delta}{p}} \frac{l_{0}}{2}\right)-\left(1+\gamma^{2}\right) \frac{\Delta l_{0}^{2}}{4 p}\right] \approx \frac{3}{\kappa a^{2}}, \\
& \rho_{2}(p)=\rho_{\mathrm{c}}\left[1-\sin ^{2}\left(\sqrt{\frac{\Delta}{p}} \frac{l_{0}}{2}\right)+\left(1+\gamma^{2}\right) \frac{\Delta l_{0}^{2}}{4 p}\right] \approx \rho_{\mathrm{c}}+\frac{3}{\kappa a^{2}} .
\end{aligned}
$$

It is clear from the above expressions that relation $\rho_{2}(p)-\rho_{1}(p)=\rho_{\mathrm{c}}$ is fulfilled. Approximations performed in (2.15) and (2.16) are valid for $p \gg \frac{\Delta l_{0}^{2}}{4} \simeq 18.9 l_{\mathrm{Pl}}^{2}$. 
Alternatively expression for the modified Friedmann equation can be written as follows

$$
H^{2}=\frac{8 \pi G_{\text {eff }}}{3} \rho-\frac{K_{\text {eff }}}{a^{2}}
$$

where effective constants are expressed as follows

$$
\begin{aligned}
& G_{\text {eff }}=G\left[1+2 \frac{\rho_{1}}{\rho_{\mathrm{c}}}-\frac{\rho}{\rho_{\mathrm{c}}}\right], \\
& K_{\text {eff }}=\frac{\kappa}{3}\left(\frac{a_{0}}{l_{0}}\right)^{2} p \frac{\rho_{1}(p) \rho_{2}(p)}{\rho_{\mathrm{c}}} .
\end{aligned}
$$

Expression for the $K_{\text {eff }}$ does not depends on the energy density $\rho$. Therefore it can be studied without specifying form of the matter. We expect that classical limit $K_{\mathrm{eff}} \rightarrow 1$ for $p \rightarrow \infty$ should be recovered. It is in fact a case, what it is clear from Fig. 1. For $p \rightarrow 0$, factor $K_{\text {eff }}$ grows and can potentially play important

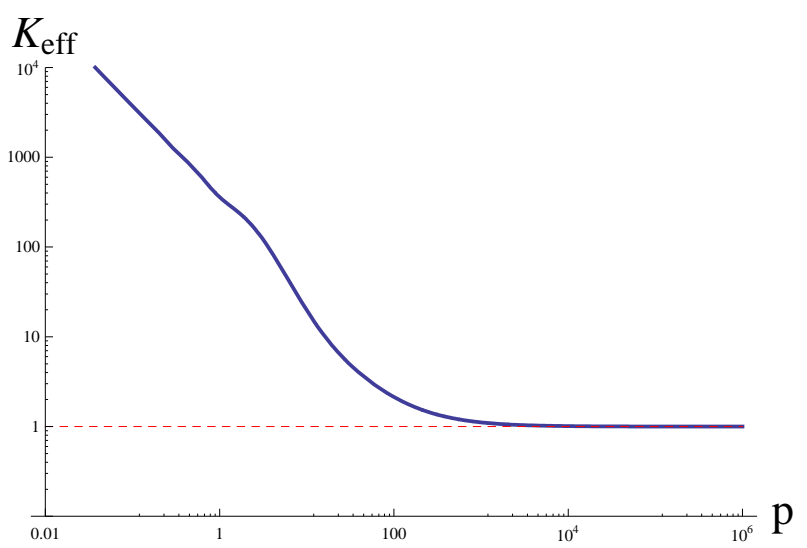

Figure 1: Effective parameter of the curvature $K_{\text {eff }}$.

role. We study this issue in the subsequent sections.

Defining energy density and pressure of the scalar field

$$
\begin{aligned}
\rho & =\frac{\dot{\phi}^{2}}{2}+V(\phi), \\
\mathcal{P} & =\frac{\dot{\phi}^{2}}{2}-V(\phi) .
\end{aligned}
$$

we can derive equation

$$
\frac{d H}{d t}=-4 \pi G\left[(\rho+\mathcal{P})-M(p) \rho_{\mathrm{c}}\right]\left[1+2 \frac{\rho_{1}}{\rho_{\mathrm{c}}}-2 \frac{\rho}{\rho_{\mathrm{c}}}\right]
$$


where to simplify notation we have defined

$$
M(p)=\left(1+\gamma^{2}\right) \frac{\Delta l_{0}^{2}}{6 p}-\frac{l_{0}}{3} \sqrt{\frac{\Delta}{p}} \sin \left(\sqrt{\frac{\Delta}{p}} \frac{l_{0}}{2}\right) \cos \left(\sqrt{\frac{\Delta}{p}} \frac{l_{0}}{2}\right) .
$$

It is worth to mention that taking $l_{0}=0$ in (2.14) and (2.22) we obtain

$$
\begin{aligned}
H^{2} & =\frac{8 \pi G}{3} \rho\left(1-\frac{\rho}{\rho_{\mathrm{c}}}\right), \\
\frac{d H}{d t} & =-4 \pi G(\rho+\mathcal{P})\left[1-2 \frac{\rho}{\rho_{\mathrm{c}}}\right]
\end{aligned}
$$

recovering the case $K=0$.

\section{Models with $\Lambda$ and free scalar field as 2D dynamical systems}

Effective loop dynamics of the flat FRW models with $\Lambda$ and free scalar field was studied in [11]. In this reference, fully analytical solutions of the model were found. Unfortunately in case of the closed model the equations become transcendental and cannot be solved analytically. Therefore other approach, to investigate dynamics, have to be applied. In our considerations we use methods of the qualitative analysis of the dynamics. Useful technique applied here is compactification of the phase space. Based on this approach it will be possible to study global properties of dynamics.

In this section we consider models with cosmological constant and free scalar field. For these models dynamics reduce to $2 \mathrm{D}$ dynamical systems, what simplify qualitative analysis. Moreover one has to remember that physical solutions are on the surface of Hamiltonian constraint $\mathcal{H}_{\text {eff }}(p, \dot{p}) \approx 0$. Therefore domain allowed for the motion reduce to one dimensional subspace. It means that on $2 \mathrm{D}$ phase plane $(p, \dot{p})$, only one trajectory represents physical motion. Other possible trajectories does not fulfil $\mathcal{H}_{\text {eff }}(p, \dot{p}) \approx 0$. It is also interesting to note that in all cases, the same maximal value of Hubble parameter $H_{\max }$ is reached. In fact this is the same $H_{\max }=\sqrt{\frac{\kappa}{12} \rho_{c}}$ as this obtained for the flat models.

\subsection{Cosmological constant}

In the first case we consider model with cosmological constant only. Energy density and pressure of the cosmological constant are given by

$$
\rho_{\Lambda}=\frac{\Lambda}{8 \pi G} \text { and } \mathcal{P}_{\Lambda}=-\frac{\Lambda}{8 \pi G} .
$$


Dynamics of the system is governed now only by modified Friedmann equation (2.14). Since left side of this equation is $H^{2} \geq 0$ on can find physical domain of motion form this condition. We plot $H^{2}$ function given by Eq. (2.14) in Fig. 2. We find that while $\rho_{\Lambda}<\rho_{c}$ then domain for the physical motion is bounded from

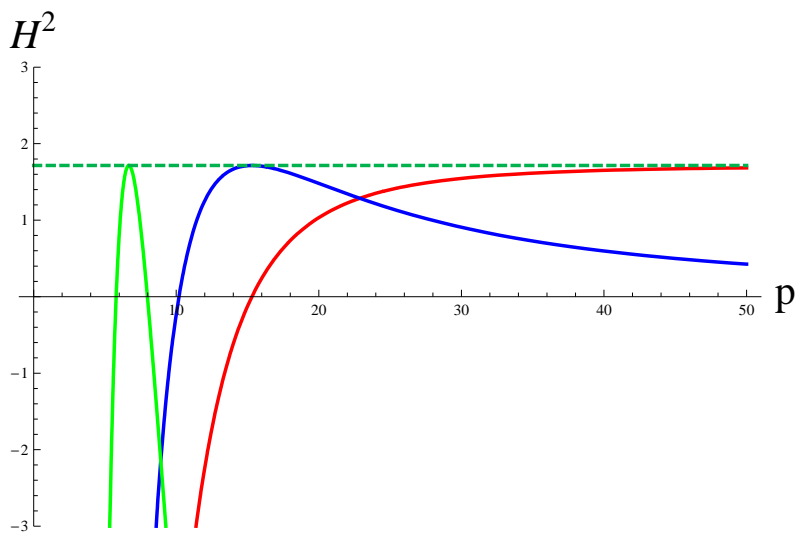

Figure 2: Square of the Hubble factor for different values of $\rho_{\Lambda}$. From right $\rho_{\Lambda}=0.5 \rho_{c}$ (red), $\rho_{\Lambda}=\rho_{c}$ (blue) and $\rho_{\Lambda}=2.5 \rho_{c}$ (green). Dashed line represents $H_{\max }^{2}=\frac{\kappa}{12} \rho_{c}$.

two sides. Therefore we expect oscillatory solutions in this regime. While $\rho_{\Lambda}=\rho_{c}$ the upper bound reach infinity. In turn, when $\rho_{\Lambda}>\rho_{c}$ upper bound disappears. It is useful to define critical value of cosmological constant

$$
\Lambda_{\mathrm{c}}=\frac{\sqrt{3} m_{\mathrm{Pl}}^{2}}{2 \pi \gamma^{3}} \simeq 21 m_{\mathrm{Pl}}^{2}
$$

since bifurcation of solutions occur when value of cosmological constant cross $\Lambda_{\mathrm{c}}$. This critical value was introduced in Ref. [11] while studying flat models in LQC. It was show there that there is no physical solutions for $\Lambda>\Lambda_{\mathrm{c}}$. In the curved models we however expect physical motion in this region.

Now we can investigate dynamics of these three cases on the phase phase portraits shown in Fig. 3. We see tree mentioned types of solutions. It is worth to stress that only thick lines represent physical trajectories. Other trajectories shown in Fig. 3 are not on the surface of Hamiltonian constraint $\mathcal{H}_{\text {eff }}(p, \dot{p}) \approx 0$. Based on approximations performed in 2.15$)$ and $(2.16)$ we find

$$
\begin{aligned}
& p_{\min } \approx \frac{3}{4} \frac{l_{0}^{2}}{\Lambda} \mapsto a_{\min } \approx \sqrt{\frac{3}{\Lambda}}, \\
& p_{\max } \approx \frac{3}{4} \frac{l_{0}^{2}}{\Lambda-\Lambda_{\mathrm{c}}} \mapsto a_{\max } \approx \sqrt{\frac{3}{\Lambda-\Lambda_{\mathrm{c}}}} .
\end{aligned}
$$



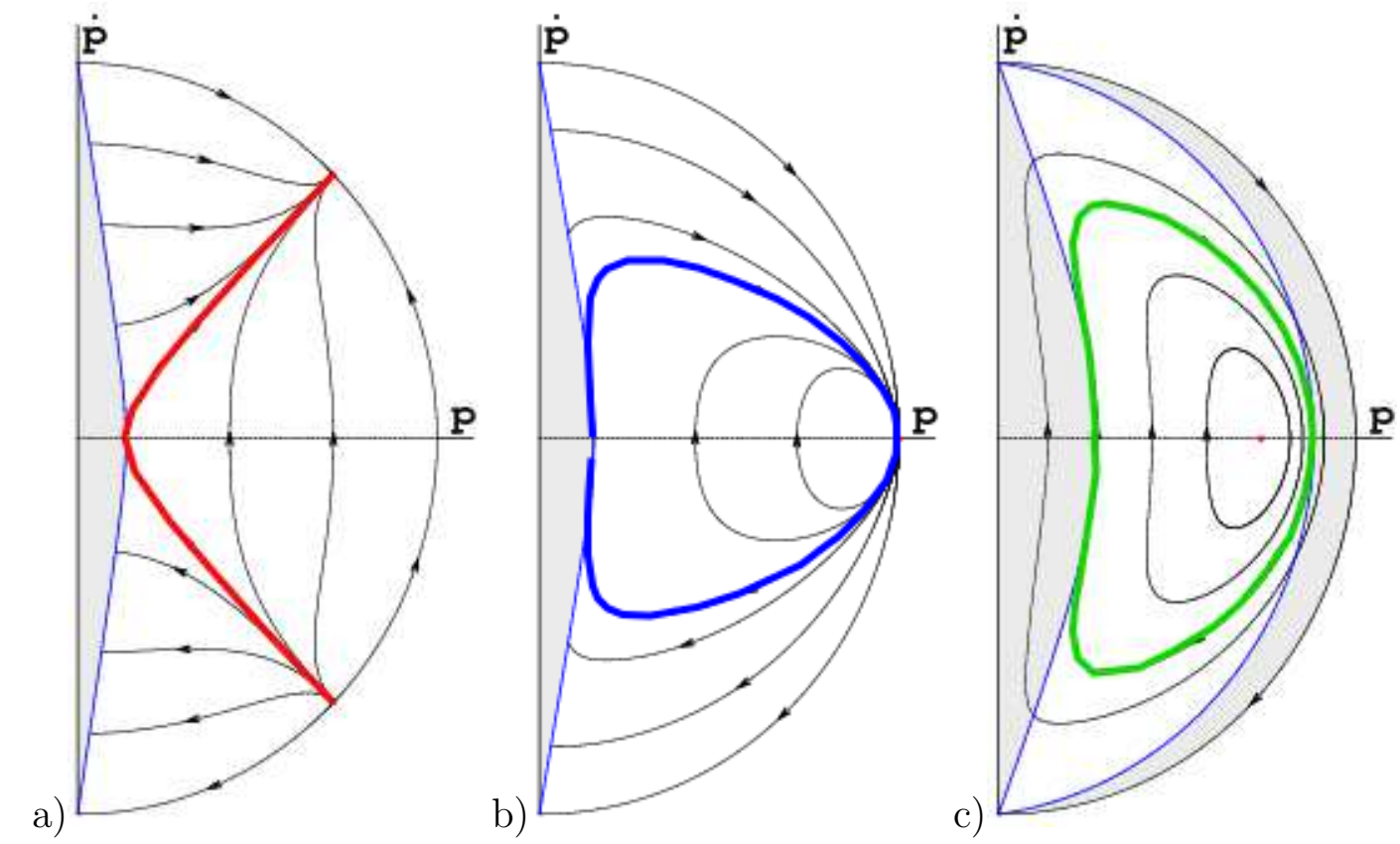

Figure 3: Phase space diagrams for the model with the cosmological constant. Shaded regions are unphysical because of $H^{2}<0$. Case a) $0<\rho_{\Lambda}<\rho_{c}$, b) $\rho_{\Lambda}=\rho_{c}$, c) $\rho_{\Lambda}>\rho_{c}$. Physical solutions, on the surface of Hamiltonian constraint $\mathcal{H}_{\mathrm{eff}}(p, \dot{p}) \approx 0$, are distinguished by thick (red, blue, green) lines.

Expression for $p_{\max }$ is however valid only for $\Lambda \geq \Lambda_{\mathrm{c}}$. When $\Lambda<\Lambda_{\mathrm{c}}$ we have $p_{\max } \rightarrow \infty$. In this case asymptotic de Sitter stage is obtained as in the flat case.

\subsection{Free scalar field}

Now we get on to example of free scalar filed which is widely used in LQC. It is due to the fact that, in this model, scalar field can be treated as internal clock. It is of great importance when purely quantum cosmological models are constructed. However it is not necessary on the semi-classical level. Therefore models with no well defined global internal time can also be studied. Example of this will be given in the subsequent section when model with self-interacting field is studied. However even in that case well defined intrinsic time can be introduced at intervals.

Considered model with free scalar filed was studied before in Ref. [6]. In this reference fully quantum analysis of the model has been done. In particular wave function for this model was computed. It was also shown that semi-classical dynamics follows precisely the quantum evolution. It is important result approv- 
ing applicability of the semi-classical approach. Here we would like to complete earlier investigations.

It is worth to remind here that energy density and pressure of the free scalar field are given by

$$
\rho_{\phi}=\frac{p_{\phi}^{2}}{2 p^{3}} \text { and } \mathcal{P}_{\phi}=\frac{p_{\phi}^{2}}{2 p^{3}}
$$

where $p_{\phi}$ is constant of integration here. Based, as earlier, on Eq. (2.14) we can find the regions $H^{2} \geq 0$. This gives us regions allowed for motion. We show exemplary curves in Fig. $ه$. It can be seen that doubly restrict region is allowed for the motion. This region is enlarged with increasing value of parameter $p_{\phi}$.

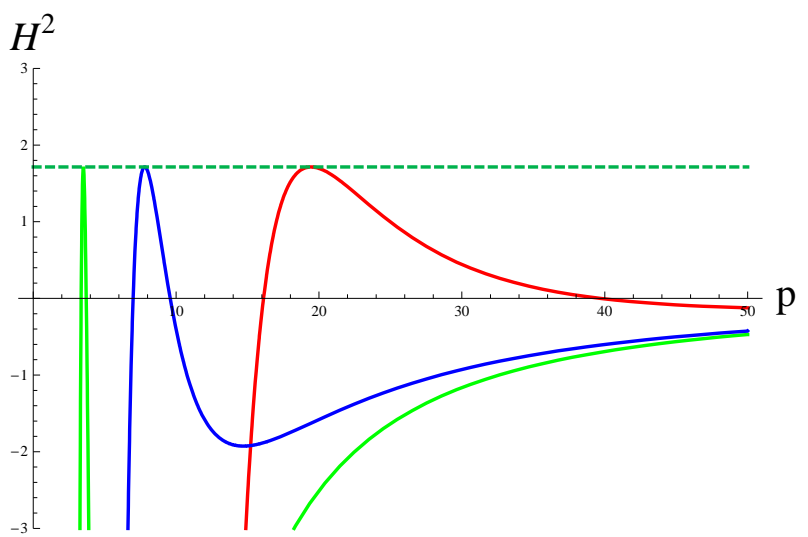

Figure 4: Square of the Hubble factor for different values of $p_{\phi}$. From left to right $p_{\phi}=20,40,100 l_{\mathrm{Pl}}$. Dashed line represents $H_{\max }^{2}=\frac{\kappa}{12} \rho_{c}$.

Based on analysis of positivity of $H^{2}$ function one expect that solutions are represented by oscillations. Namely universe bouncing between two edges of the region allowed for motion. It can be approved looking on phase portrait for this system, shown in Fig. 5. In fact physical solution is represented by closed curve. Similarly like in the previous case, physical solution lie on the surface of Hamiltonian constraint. Here we can also find

$$
p_{\min } \approx \sqrt[3]{\frac{p_{\phi}^{2}}{2 \rho_{\mathrm{c}}}} \text { and } p_{\max } \approx \sqrt{\frac{\kappa}{3} \frac{2}{l_{0}^{2}} p_{\phi}^{2}}
$$

based on approximations performed in (2.15) and (2.16). These approximations are valid for $p \gg \frac{\Delta l_{0}^{2}}{4} \simeq 18.9 l_{\mathrm{Pl}}^{2}$. 


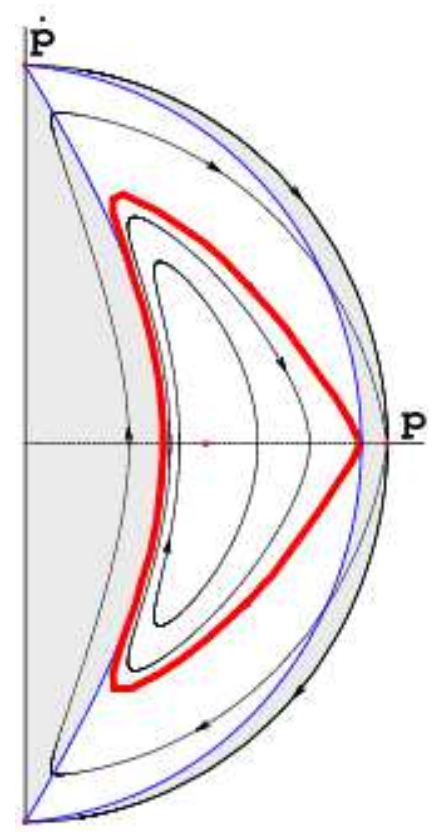

Figure 5: Phase space diagram for the model with free scalar field. The shaded regions are unphysical because $H^{2}<0$ there. Physical solution, on the surface of Hamiltonian constraint $\mathcal{H}_{\mathrm{eff}}(p, \dot{p}) \approx 0$, is distinguished by thick (red) line.

\section{Qualitative analysis of the model with quadratic poten- tial function}

Self-interacting scalar fields are commonly used in the modern cosmology. It comes from the fact that violation of the strong energy condition $\rho+3 p \geq 0$ can be obtained with them. Namely $\rho_{\phi}+3 p_{\phi}=2\left(\dot{\phi}^{2}-V(\phi)\right)$ and it is possible to have $V(\phi)>\dot{\phi}^{2}$ what leads to $\rho+3 p<0$. In consequence accelerating stage of the evolution of the universe occurs. This mechanism can be therefore applied to modelling dynamics of the inflation or present era of dark energy. Here we concentrate on this former. It is due to the fact that stage of inflation is proceeded by the Planck era and both phases should be dynamically related. Namely initial conditions for the inflation should be given in the Planck epoch. In our approach the Planck epoch is described by the closed loop cosmology. It is interesting to see how phase of inflation emerge in this scenario. In fact this was already studied in case of the flat model [10]. It was shown that phase of bounce can be naturally connected with the inflation. Moreover inflationary attractor is generic and is realised for the broad range of the initial conditions. Here we are going to check whether curvature can affect this scenario. 
In our considerations we assume that considered scalar field is massive. Then energy density is given by

$$
\rho_{\phi}=\frac{1}{2} \dot{\phi}^{2}+V(\phi)=\frac{1}{2} \dot{\phi}^{2}+\frac{1}{2} m^{2} \phi^{2} .
$$

Dynamical system, in case of self-interacting scalar fields, can be written in the form

$$
\begin{aligned}
\dot{\phi} & =y, \\
\dot{y} & =-\frac{3}{2} \frac{z}{p} y-\frac{d V(\phi)}{d \phi}, \\
\dot{p} & =z \\
\dot{z} & =2 \frac{\kappa}{3} \rho_{c} p\left\{2\left(\sin ^{2}\left(\sqrt{\frac{\Delta}{p}} \frac{l_{0}}{2}\right)-\left(1+\gamma^{2}\right) \frac{\Delta l_{0}^{2}}{4 p}+\frac{1}{\rho_{c}}\left(\frac{1}{2} y^{2}+V(\phi)\right)\right) \times\right. \\
& \times\left(\cos ^{2}\left(\sqrt{\frac{\Delta}{p}} \frac{l_{0}}{2}\right)+\left(1+\gamma^{2}\right) \frac{\Delta l_{0}^{2}}{4 p}-\frac{1}{\rho_{c}}\left(\frac{1}{2} y^{2}+V(\phi)\right)\right) \\
& -\frac{1}{2}\left(\frac{1}{2} \sqrt{\frac{\Delta}{p}} l_{0} \sin \left(\sqrt{\frac{\Delta}{p}} l_{0}\right)-\left(1+\gamma^{2}\right) \frac{\Delta l_{0}^{2}}{2 p}+\frac{3}{\rho_{c}} y^{2}\right) \times \\
& \left.\times\left(\cos \left(\sqrt{\frac{\Delta}{p}} l_{0}\right)+\left(1+\gamma^{2}\right) \frac{\Delta l_{0}^{2}}{2 p}-\frac{2}{\rho_{c}}\left(\frac{1}{2} y^{2}+V(\phi)\right)\right)\right\}
\end{aligned}
$$

together with the constrain

$$
\frac{1}{4} z^{2}=\frac{\kappa}{3} \rho_{c} p^{2}\left(\sin ^{2}\left(\sqrt{\frac{\Delta}{p}} \frac{l_{0}}{2}\right)-\left(1+\gamma^{2}\right) \frac{\Delta l_{0}^{2}}{4 p}+\frac{\rho_{\phi}}{\rho_{c}}\right)\left(\cos ^{2}\left(\sqrt{\frac{\Delta}{p}} \frac{l_{0}}{2}\right)+\left(1+\gamma^{2}\right) \frac{\Delta l_{0}^{2}}{4 p}-\frac{\rho_{\phi}}{\rho_{c}}\right) .
$$

The condition of the positivity of this expression implies that for a constant value of $p$ the motion of the dynamical system in the phase plane $(\phi, \dot{\phi})$ is restricted to

$\rho_{c}\left(\left(1+\gamma^{2}\right) \frac{\Delta l_{0}^{2}}{4 p}+\cos ^{2}\left(\sqrt{\frac{\Delta}{p}} \frac{l_{0}}{2}\right)\right) \geq \frac{1}{2} y^{2}+\frac{1}{2} m^{2} \phi^{2} \geq \rho_{c}\left(\left(1+\gamma^{2}\right) \frac{\Delta l_{0}^{2}}{4 p}-\sin ^{2}\left(\sqrt{\frac{\Delta}{p}} \frac{l_{0}}{2}\right)\right)$

In Fig. 6 this region is represented by the shaded field (in this example $p=10$ ). For a given time region allowed for the motion is in the form of a ring. External edge is due to quantum gravity effects while internal one coming from curvature. Radius of internal edge decreases for increasing $p$. In Fig. 6 we show exemplary trajectories on the $(\phi, \dot{\phi})$ phase portrait. Two general types of trajectories are 
shown there. First type of trajectories is initialised in the point of maximal displacement of the scalar field in the potential, then $\dot{\phi}=0$. Then filed falls down through the intermediate slow-roll regime, leading to the phase of inflation. This phase of inflation is seen as nearly horizontal attractor line in Fig. 6. It can be seen also trajectories starting from lower displacements are directed towards this inflationary attractor. These trajectories are shown also in Fig. 7 where corresponding evolution on the $(\phi, p)$ plane is presented.

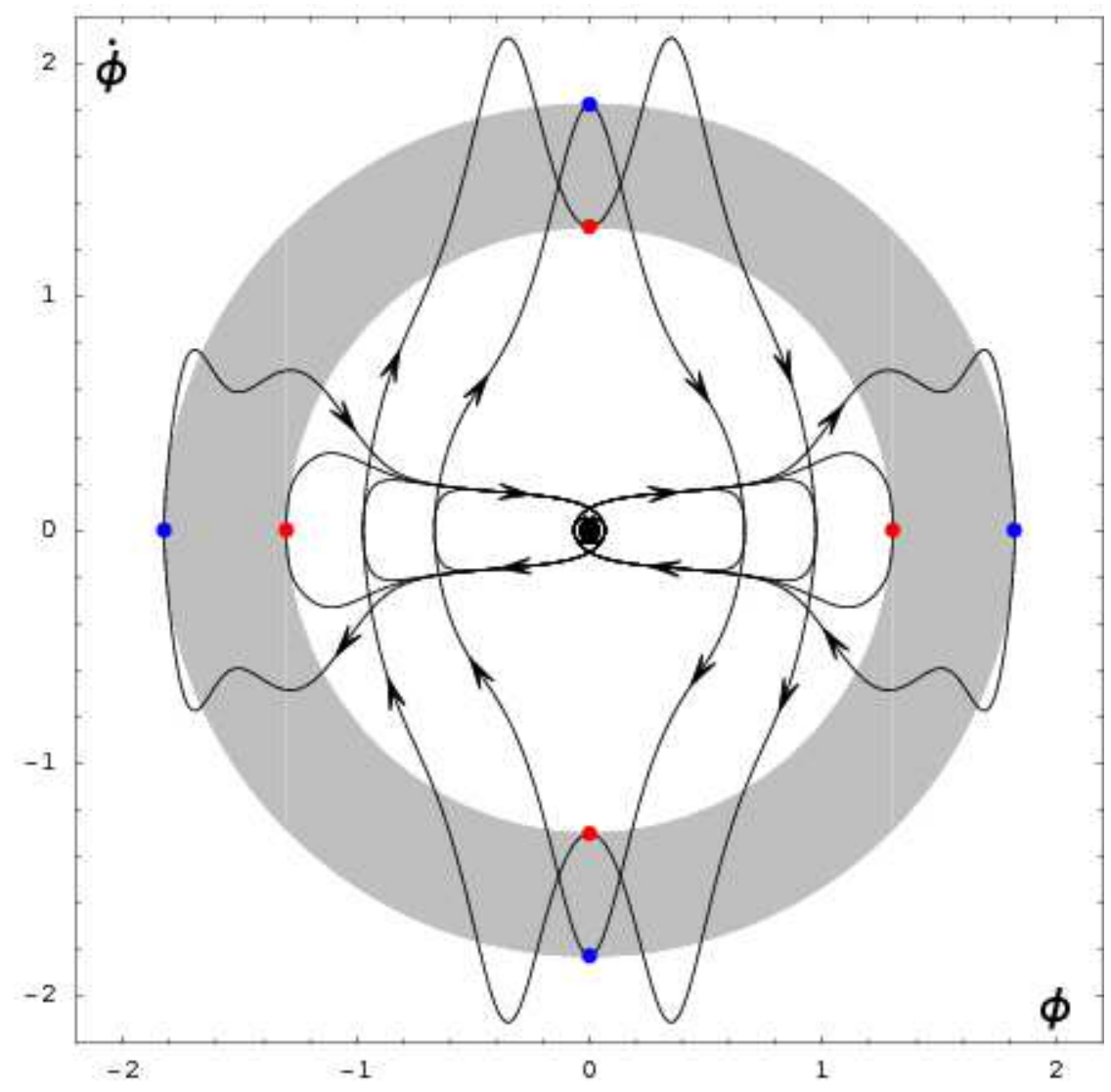

Figure 6: Projection of the $3 D$ phase space diagram for the model with the quadratic potential function and $m^{2}=1$ on the $(\phi, \dot{\phi})$ plane. The shaded field denotes region admissible for motion for constant value of $p=10$, size of this region depends on the value of $p$. The red and blue dots represent location of the initial conditions taken for numerical simulations.

Trajectories of the second kind are initialised in the bottom of the potential, where $\phi=0$. These trajectories are also directed to the same inflationary attractor. It is worth to analyse this behaviour in more details. Considered evolution 
is represented by the curves starting at $\phi=0$ in Fig. 7. Here initial state is

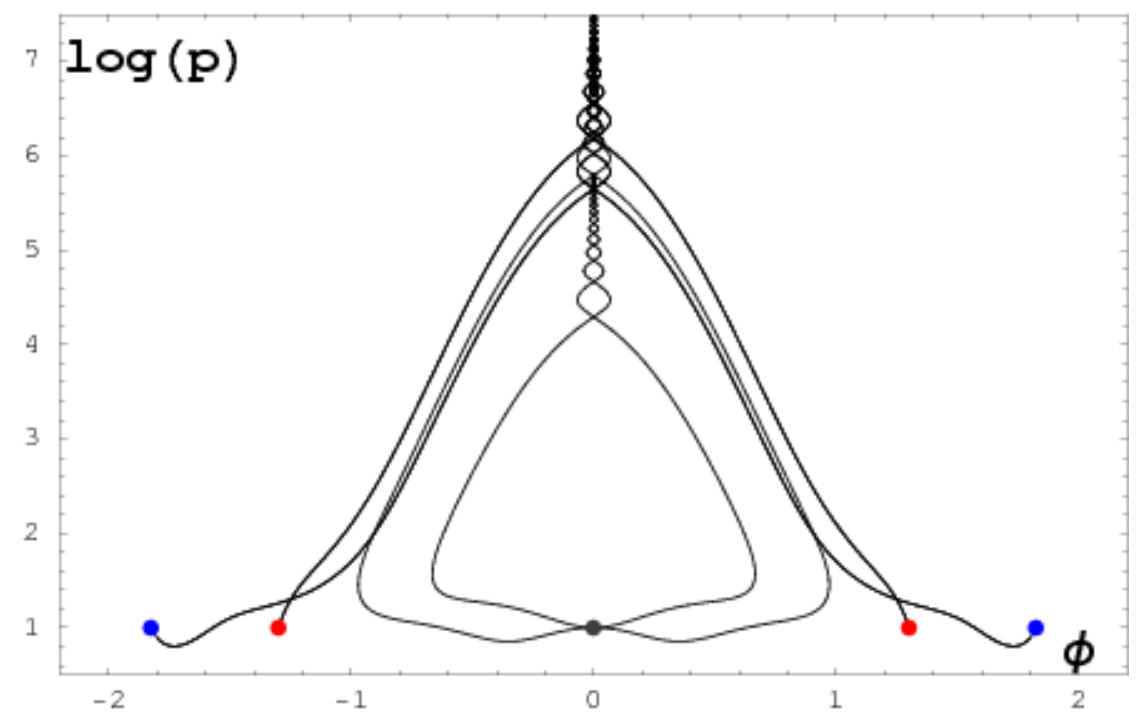

Figure 7: Projection of the $3 D$ phase space diagram for the model with the quadratic potential function and $m^{2}=1$ on the $(\phi, \log (p))$ plane. The arrows on the trajectories are omitted due to symmetries (every line can represent both expanding or contracting phase of evolution).

given in the contracting pre-bounce universe. Field is initially in the bottom of the potential well. In this stage, value of the Hubble factor is neglected with respect to $m^{2}$. Therefore field undergoes certain oscillations. It can be seen that is this stage field behaves like a dust matter. Universe behaves therefore like contracting, matter dominated one. However when bounce is approached then $H$ grows and becomes significant for the dynamics of $\phi$. Since $\mathrm{H}$ is negative act as anti-friction term in equation of motion

$$
\ddot{\phi}+3 H \dot{\phi}+m^{2} \phi=0
$$

driving $\phi$ up the potential well. For the considered trajectory, field is driven up to $\phi \simeq \pm 1$ in Planck units. Then typical slow-roll inflation takes place leading to exponential growth of $p$. Field, damped by the friction term, falls down the potential well and starts to oscillate finally. Then again matter-like stage occur. However in this point phase of reheating should stars. This phase however cannot be studied without extension of this model. Therefore physical analysis of dynamics should be stopped at this point. 


\section{Remarks on inverse volume corrections}

In the previous sections we have considered models with quantum holonomy corrections. Here we are making some remarks on the possible modifications due to the so called inverse volume corrections. In the flat models definition of these corrections is ambiguous since it deepens on the fiducial cell volume. However in the considered closed model, fiducial cell volume is fixed and inverse volume corrections are well defined. Since effects of inverse volume corrections were extensively studied in literature we are not going to perform full analysis of them. Interested reader can find more information abut them in Ref. [20, [0]. In particular interesting feature of the inverse volume corrections is the phase of super-inflation [21].

Here we are going to show where effects of inverse volume corrections start to be important. We begin this analysis with general effective Hamiltonian derived in Ref. [6]

$$
\begin{aligned}
\mathcal{H}_{\mathrm{eff}} & =\frac{A(v)}{16 \pi G}\left[\sin ^{2}\left(\bar{\mu} c-\bar{\mu} \frac{l_{0}}{2}\right)-\sin ^{2}\left(\bar{\mu} \frac{l_{0}}{2}\right)+\left(1+\gamma^{2}\right) \frac{\bar{\mu}^{2} l_{0}^{2}}{4}\right] \\
& +\left(\frac{8 \pi G \gamma}{6}\right)^{-3 / 2} B(v) \frac{p_{\phi}^{2}}{2} .
\end{aligned}
$$

Here functions $A(v)$ and $B(v)$ contains inverse volume corrections and are given by

$$
\begin{aligned}
& A(v)=-\frac{27 K}{4 \gamma^{3 / 2}} \sqrt{\frac{8 \pi}{6}}|v||| v-1|-| v+1||, \\
& B(v)=\left(\frac{3}{2}\right)^{3} K|v||| v+\left.1\right|^{1 / 3}-\left.|v-1|^{1 / 3}\right|^{3}
\end{aligned}
$$

where

$$
K=\frac{2 \sqrt{2}}{3 \sqrt{3 \sqrt{3}}} .
$$

For $v \gg 1$ functions can be expanded as follows

$$
\begin{aligned}
& A(v) \simeq-\frac{2 \sqrt{48 \pi} v}{\gamma^{3 / 2} 3 \sqrt{3} K}, \\
& B(v) \simeq \frac{K}{v} .
\end{aligned}
$$

We show both functions (5.2) and (5.3) with corresponding approximations (5.5) and (5.6) in Fig. 8. Approximations (5.5) and (5.6) are precisely those applied 

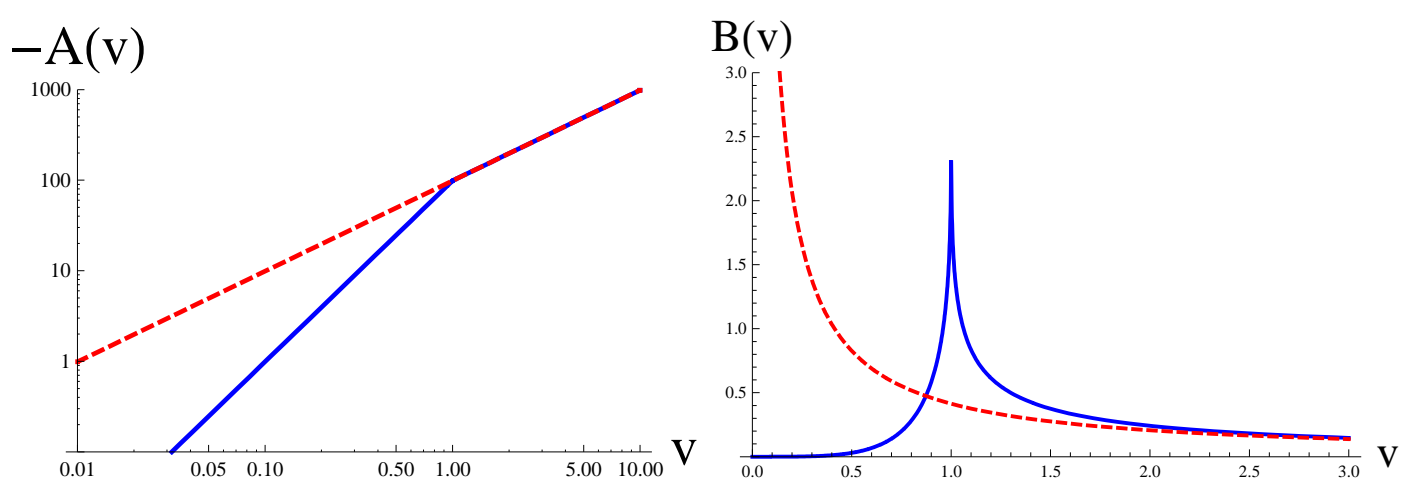

Figure 8: Left : Inverse volume correction $A(v)$ (straight line) together with approximation used in this paper (dashed line). Right : Inverse volume correction $B(v)$ (straight line) together with approximation used in this paper (dashed line).

in this paper, leading to initial Hamiltonian (2.1). Form Fig. 8 we see that corrections become important below $v \approx 2$. Since relation between $p$ and $v$ variables is given by

$$
p(v)=\frac{\kappa \gamma}{6}\left(\frac{v}{K}\right)^{2 / 3}
$$

we find $p(2) \simeq 2.8$. Therefore for $p \lesssim 3$ inverse volume corrections becomes significant. So we have to be aware that results obtained in this paper are valid for $p \gtrsim 3$ and below this value other quantum effects should take place. However only small fraction of trajectories probe this region. In fact we can always choose sufficient initial conditions to avoid this region. For instance for the model with a free scalar field, sufficiently high value of $p_{\phi}$ has to be chosen. Namely condition for $p>3$ gives $p_{\phi}>18 l_{\mathrm{Pl}}$.

\section{Summary}

In the classical cosmology positive curvature introduces $-1 / a^{2}$ term in the Friedmann equation. In the early universe other terms like radiation $1 / a^{4}$ dominate and dynamical effect of curvature is negligible. However also quantum gravitational effects start to be important then and dynamics should be modified. Loop quantum cosmology gives us opportunity to study these effects. In particular in the effective formulation of LQC dynamics is governed by the classical equations with proper quantum corrections. These corrections modify also the curvature term $-1 / a^{2}$ and therefore dynamical significance of this term can be changed in the quantum regime. In this paper we have investigated whether curvature term could play significant role in early universe due to the quantum effects. We 
have found that curvature does not affect evolution in the quantum regime. In particular, maximal value of the Hubble factor is the same like in the flat case, namely $H_{\max }=\sqrt{\frac{\kappa}{12} \rho_{c}}$.

In our considerations we concentrated on the models with cosmological constant, free scalar field and massive scalar field. In the models with cosmological constant and free scalar field, dynamics reduce to 2D system. For the free field case, universe undergoes non-singular oscillations. This behaviour does not depend on the value of parameter $p_{\phi}$. However for the model with cosmological constant only, type of solution depends on the value of $\Lambda$. For $\Lambda<\Lambda_{\mathrm{c}}$ bouncing solutions with asymptotic de Sitter stages are obtained. While $\Lambda>\Lambda_{\mathrm{c}}$ we find non-singular oscillations. Case $\Lambda=\Lambda_{\mathrm{c}}$ is intermediate state when upper boundary

reaches infinity. Here $\Lambda_{c}$ is critical value of $\Lambda$ and is given by $\Lambda_{\mathrm{c}}=\frac{\sqrt{3} m_{\mathrm{Pl}}^{2}}{2 \pi \gamma^{3}} \simeq 21 m_{\mathrm{Pl}}^{2}$. Emergence of this value is due to quantum modifications of the dynamics. Therefore observed change of the type of solution is of the quantum origin and does not appear in the classical case.

Physically interesting consequences occur in the models with self-interacting scalar field. Namely, the point is that, bounce gives mechanism to set good initial conditions for inflation. During the bounce field is driven up the potential well what is necessary to start phase of inflation. In the classical theory of inflation initial conditions have to be fixed suitably. Here they can be obtained from the different pre-bounce evolutions without need of any precise adjustment. This is nice feature of the bounce stage and is now confirmed for the flat and closed models in LQC.

\section{References}

[1] A. Ashtekar and J. Lewandowski, Class. Quant. Grav. 21 (2004) R53 [arXiv:gr-qc/0404018].

[2] M. Bojowald, Living Rev. Rel. 11 (2008) 4.

[3] A. Ashtekar, T. Pawlowski and P. Singh, Phys. Rev. Lett. 96 (2006) 141301 [arXiv:gr-qc/0602086].

[4] A. Ashtekar, T. Pawlowski and P. Singh, Phys. Rev. D 73 (2006) 124038 [arXiv:gr-qc/0604013].

[5] A. Ashtekar, T. Pawlowski and P. Singh, Phys. Rev. D 74 (2006) 084003 [arXiv:gr-qc/0607039]. 
[6] A. Ashtekar, T. Pawlowski, P. Singh and K. Vandersloot, Phys. Rev. D 75 (2007) 024035 [arXiv:gr-qc/0612104].

[7] L. Szulc, W. Kaminski and J. Lewandowski, Class. Quant. Grav. 24 (2007) 2621 [arXiv:gr-qc/0612101].

[8] K. Vandersloot, Phys. Rev. D 75 (2007) 023523 [arXiv:gr-qc/0612070].

[9] L. Szulc, Class. Quant. Grav. 24 (2007) 6191 [arXiv:0707.1816 [gr-qc]].

[10] P. Singh, K. Vandersloot and G. V. Vereshchagin, Phys. Rev. D 74 (2006) 043510 [arXiv:gr-qc/0606032].

[11] J. Mielczarek, T. Stachowiak and M. Szydlowski, Phys. Rev. D 77 (2008) 123506 [arXiv:0801.0502 [gr-qc]].

[12] D. W. Chiou, Phys. Rev. D 76 (2007) 124037 [arXiv:0710.0416 [gr-qc]].

[13] D. W. Chiou, arXiv:0812.0921 [gr-qc].

[14] P. Malkiewicz and W. Piechocki, arXiv:0903.4352 [gr-qc].

[15] P. Dzierzak, J. Jezierski, P. Malkiewicz and W. Piechocki, arXiv:0810.3172 [gr-qc].

[16] K. A. Meissner, Class. Quant. Grav. 21 (2004) 5245 [arXiv:gr-qc/0407052].

[17] J. Mielczarek and M. Szydlowski, Phys. Rev. D 77 (2008) 124008 [arXiv:0801.1073 [gr-qc]].

[18] O. Hrycyna, J. Mielczarek and M. Szydlowski, arXiv:0804.2778 [gr-qc].

[19] L. F. Li and J. Y. Zhu, arXiv:0812.3552 [gr-qc].

[20] K. Vandersloot, Phys. Rev. D 71 (2005) 103506 [arXiv:gr-qc/0502082].

[21] E. J. Copeland, D. J. Mulryne, N. J. Nunes and M. Shaeri, Phys. Rev. D 77 (2008) 023510 [arXiv:0708.1261 [gr-qc]]. 